\title{
PROVE IDRAULICHE SU UN SEMOVENTE IRRIGUO
}

\author{
Giuseppe Taglioli, Angelo Fabbri
}

\section{INTRODUZIONE}

\section{Introduction}

L'irrigazione rappresenta uno dei fattori determinanti per incrementare e soprattutto per stabilizzare le rese produttive delle colture sia erbacee che arboree. Anche nei climi sub-umidi, nelle annate in cui le precipitazioni naturali sono insufficienti durante le fasi vegetative più significative, senza ricorrere all'intervento irriguo vengono vanificati per numerose colture, a causa di un'inadeguata produzione quanti-qualitativa, i vantaggi derivanti dal corretto ed oneroso utilizzo di altri fattori della produzione (concimazioni, sementi selezionate, trattamenti fitosanitari, lavorazioni meccaniche).

L'irrigazione, come tutti i fattori della produzione, è comunque soggetta alle leggi economiche della produttività (differenza benefici-costi): il suo costo d'esercizio, legato anche al metodo irriguo utilizzato, a volte può infatti essere superiore alla maggiore resa produttiva indotta dall'irrigazione stessa. Mentre per le colture arboree il ricorso all'irrigazione, anche per l'aspetto qualitativo, rappresenta un vantaggio economico in ogni ambiente pedoclimatico, per quelle erbacee, specie in ambienti sub-umidi, tale ricorso pone spesso l'interrogativo sulla sua validità economica [4].

Tra i metodi irrigui utilizzabili, quelli gravitazionali come l'infiltrazione o lo scorrimento, ancora molto utilizzati in diverse zone geografiche italiane (grazie anche al sistema tariffario dell'acqua "a superficie"), comportano un minor costo d'esercizio ma presentano anche diversi aspetti problematici: necessità di grandi

Memorie presentate il 30.03.2005; accettato il 09.11.2007

1 Dott. Giuseppe TAglioli, professore associato; ing. Angelo FABBRI, ricercatore confermato - Dipartimento di Economia e Ingegneria Agrarie, Università degli Studî di Bologna (www.agrsci.unibo.it/ deiagra; Viale G.Fanin, 50 - 40127 Bologna). Il dott. Taglioli ha ideato, progettato e condotto la sperimentazione. L'ing. Fabbri ha definito la parte teorica concernente il miglior posizionamento degli irrigatori. Nel contesto dell'attività qui relazionata sono stati utilizzati fondi MIUR 60\%. portate, elevato consumo idrico legato alla bassa efficienza ed alle caratteristiche della loro gestione, disomogeneità distributiva, necessità di contenimento delle dimensioni delle singole unità irrigue, impiego di manodopera più specializzata. $[2,5]$.

Soprattutto sotto l'aspetto del risparmio idrico ed energetico, l'attuale tendenza mira a favorire la trasformazione degli impianti gravitazionali nella tipologia in pressione (aspersione e microirrigazione). In tale ottica un'attenzione particolare viene oggi rivolta ai semoventi irrigui, in particolare del tipo ad ala avvolgibile (commercialmente noti come "rotoloni") con irrigatore unico d'estremità in quanto, specie per le colture erbacee, largamente diffusi in diverse realtà agricole non solo italiane. Tale diffusione è come noto dovuta a molti aspetti positivi, sia tecnici che operativi, nonostante esistano tuttora alcuni specifici problemi, non solo di tipo economico, che pongono interrogativi sulla validità generale di tali soluzioni irrigue $[8,9]$.

\section{OBIETTIVI DELLA RICERCA}

\section{Aim of the research}

Al fine di fornire un contributo alla conoscenza delle caratteristiche tecnico-economiche di questi diffusissimi semoventi irrigui, anche a fronte della scarsità di informazioni di letteratura, è proseguita una ricerca interdisciplinare, già da tempo avviata, che ha potuto fornire indicazioni sostanzialmente su tre aspetti:

1) idraulico-tecnologici ( prestazioni del rotolone);

2) agronomici (risposta produttiva del mais all'irrigazione di alcune tesi irrigue e non);

3) economici (convenienza economica dell'irrigazione: differenza benefici-costi).

Con la presente relazione vengono riferiti in dettaglio i risultati di natura idraulica mentre l'aspetto economico, qui trattato in alcuni suoi punti, sarà oggetto insieme a quello agronomico di una successiva comunicazione $[6,7]$. 


\section{MATERIALI E METODI}

\section{Material and methods}

Le prove idrauliche sono state eseguite nell'estate 2001 utilizzando un semovente ad ala avvolgibile con irrigatore unico d'estremità (di seguito denominato più semplicemente "rotolone") presso l'azienda agricola Calanca, in comune di Mirandola in provincia di Modena, caratterizzata da un terreno di tipo argilloso. Il "rotolone" utilizzato è un modello Fer-Bo 90 a turbina, con 300 metri di tubo, non provvisto di regolarizzatore della velocità, alimentato da un sistema pompatrattrice (pompa centrifuga Rovatti da $30 \mathrm{~kW}$ e trattrice Fiat 1000 Super $73 \mathrm{~kW}$ ).

Le prove di irrigazione sono state effettuate su un appezzamento di circa 300 metri di lunghezza per una larghezza bagnata di circa $90 \mathrm{~m}$, sul quale sono stati disposti 55 pluviometri, secondo lo schema di figura 1 .

Nella stessa figura sono anche indicate le basi utilizzate per il calcolo della velocità di lavoro e la collocazione dell'anemometro. Tale disposizione, pur senza fare riferimento diretto ad alcuno specifico standard, risponde all'esigenza di disporre di un numero di punti di rilievo, giudicato sufficiente sulla base di un'attività ventennale nel settore $[1,3]$.

$\mathrm{Al}$ fine di valutare l'influenza della uniformità della velocità di avanzamento sulla distribuzione sono state effettuate due prove differenti. Nella prima si è utilizzato il semovente senza alcun sistema di regolarizzazione della velocità mentre nella seconda si è intervenuto, manualmente, sul variatore di velocità per correggere tratto per tratto il valore della velocità di lavoro al valore desiderato.

Entrambe le prove sono state effettuate con un boccaglio sull'irrigatore di diametro $27,6 \mathrm{~mm}$, pressione alla turbina di $0,8 \mathrm{MPa}$, pressione all'irrigatore di 0,4 $\mathrm{MPa}$, portata di $16 \mathrm{l} / \mathrm{s}$, velocità di lavoro di $25,6 \mathrm{~m} / \mathrm{h}$ in modo da ottenere un valore di altezza media d'adacquamento di $25 \mathrm{~mm}$ sui circa 90 metri di larghezza utile bagnata indicata dal costruttore.

$\mathrm{Si}$ è anche provveduto a fare funzionare da fermo l'irrigatore nelle due posizioni all'inizio e alla fine del percorso con un tempo di sosta $t s=0,285 \mathrm{tr}$ (essendo $t r$ è il tempo che impiega l'irrigatore per percorrere una distanza pari alla gittata) necessario per distribuire anche in queste due zone una quantità sufficiente di acqua rispetto alla zona di regime [5].

I valori delle gittate e delle pressioni riscontrati nelle prove sono state confrontati con quelli indicati dalla ditta costruttrice. Velocità e direzione del vento sono state controllate attraverso un anemometro.

Sono stati rilevati anche i consumi energetici del sistema di pompaggio e la manodopera impiegata per gli spostamenti e gli allestimenti del semovente.

La valutazione della qualità dell'adacquamento ha riguardato il grado di polverizzazione del getto, la determinazione dei valori dell'intensità oraria di precipitazione (Ih $=Q / 2 \cdot R^{2} ; Q$, portata; $R$, gittata) che, per una corretta distribuzione idrica, non dovrebbe eccedere il

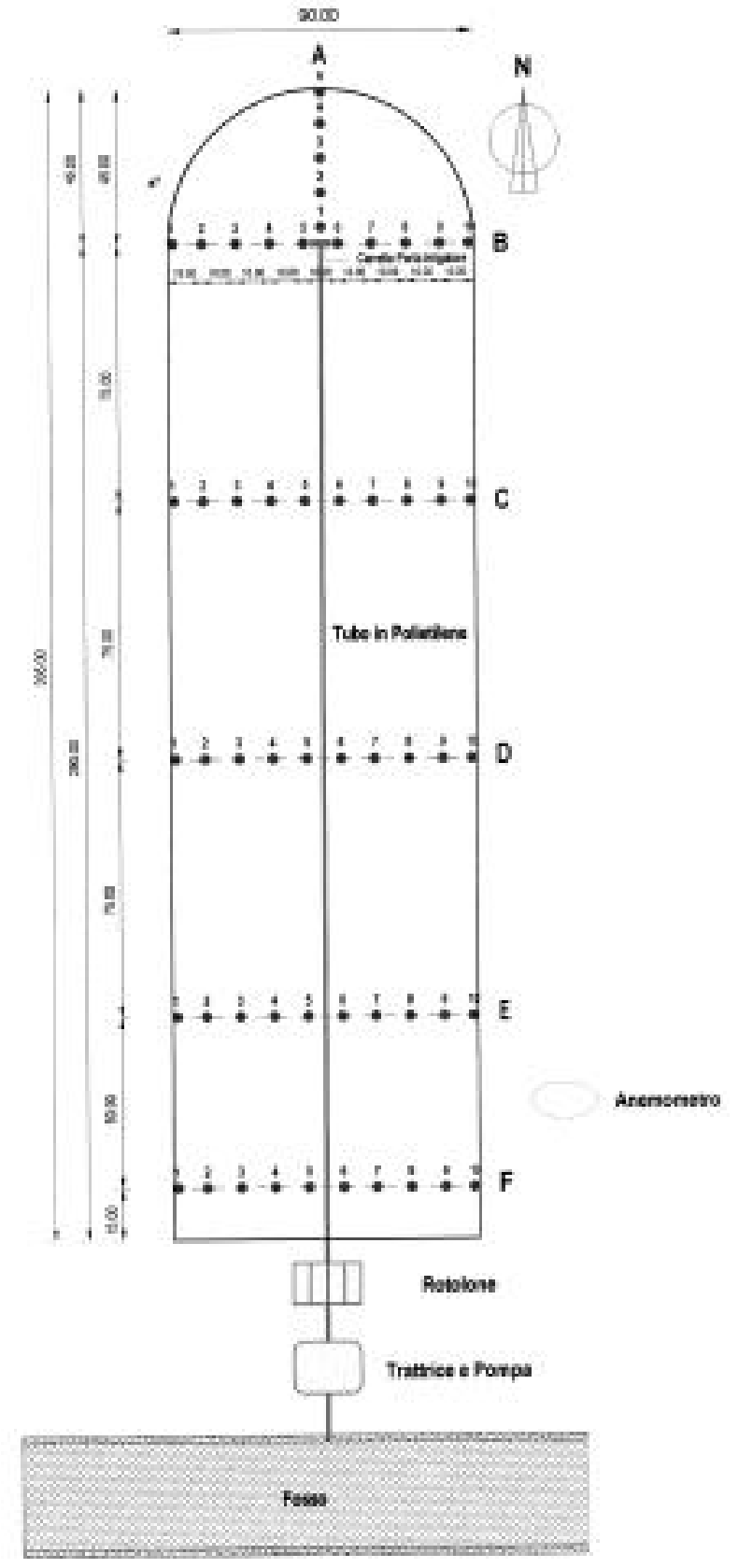

Fig. 1 - Schema della posizione dei pluviometri sul campo prova. Fig. 1 - Sketch of the positioning of the rain gauges on the test field.

coefficiente di permeabilità del terreno. L'adeguatezza della polverizzazione è valutata attraverso un indice di efficienza Ie corrispondente al rapporto tra la gittata e la pressione, e un indice di polverizzazione Ip corrispondente al rapporto tra la pressione e il diametro del boccaglio all'irrigatore (tabella $1,[10]$ ).

Le due prove tecnico-idrauliche hanno permesso quindi di ottenere informazioni relative ai seguenti parametri:

- distribuzione pluviometrica nell'area bagnata;

- velocità di lavoro;

- rispondenza di gittate e pressioni;

- qualità dell'adacquamento;

- consumi energetici e impiego di manodopera. 


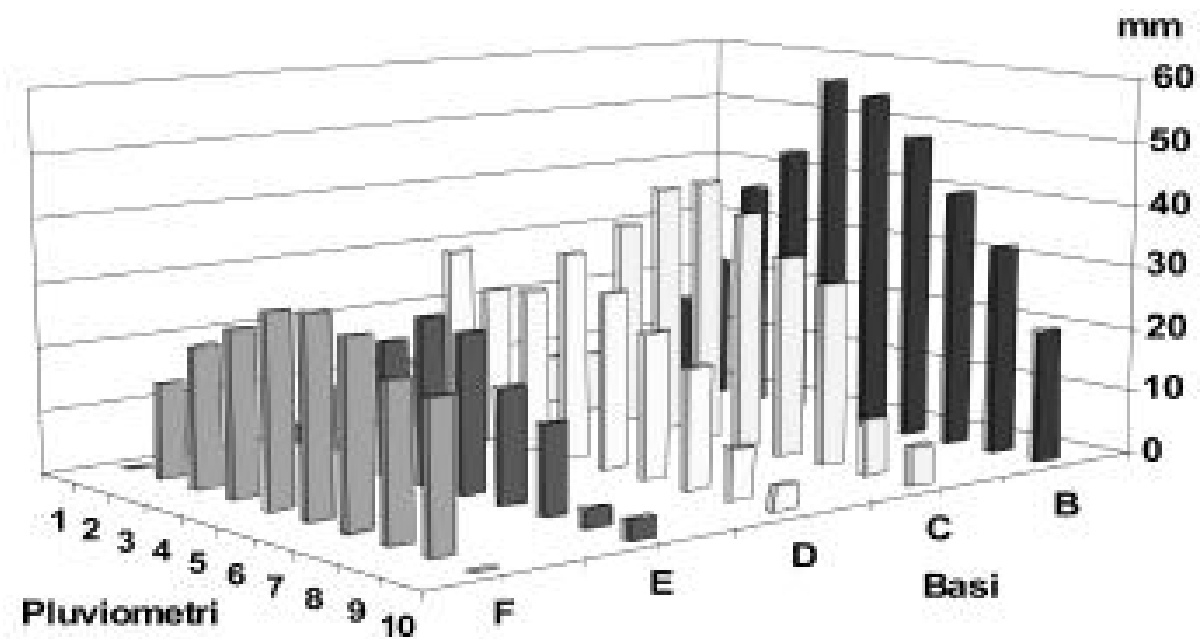

Fig. 2 - Andamento generale della pluviometria.

Fig. 2 - Overall rainfall value.

\begin{tabular}{|c|c|c|l|}
\hline Classe & \multicolumn{2}{|c|}{ Indice } & \\
\hline $\boldsymbol{a}-\mathbf{1}$ & $1,1-1,0$ & $1,02-1,41$ & $\begin{array}{l}\text { pioggia molto grossa, per } \\
\text { erbai e terreni sabbiosi }\end{array}$ \\
\hline $\boldsymbol{a}-\mathbf{2}$ & $0,99-0,91$ & $1,54-1,96$ & $\begin{array}{l}\text { pioggia grossa per erbai e } \\
\text { terreni sabbiosi }\end{array}$ \\
\hline $\boldsymbol{b}-\mathbf{1}$ & $0,88-0,78$ & $2,13-2,56$ & $\begin{array}{l}\text { pioggia media, per colture } \\
\text { erbacee e terreni medio } \\
\text { argillosi }\end{array}$ \\
\hline $\boldsymbol{b}-\mathbf{2}$ & $0,75-0,66$ & $2,70-3,23$ & $\begin{array}{l}\text { pioggia fine, per colture } \\
\text { erbacee e terreni medio } \\
\text { argillosi }\end{array}$ \\
\hline $\boldsymbol{c}$ & $<0,63$ & $>3,33$ & $\begin{array}{l}\text { pioggia molto fine, per } \\
\text { semenzai, } \\
\text { floricoltura,terreni molto } \\
\text { argillosi }\end{array}$ \\
\hline
\end{tabular}

TABELla 1 - Valori della polverizzazione del getto in funzione del tipo di terreno e colture. [14]

TABLE 1 - Recommended spray values vs. crop and soil type.

\begin{tabular}{|c|c|c|c|}
\hline $\begin{array}{c}\text { posizione } \\
\text { delle basi } \\
\text { di velocità }\end{array}$ & $\begin{array}{c}\text { distanza } \\
(\mathrm{m})\end{array}$ & $\begin{array}{c}\text { velocità media- } \\
1^{\mathrm{a}} \text { prova } \\
(\mathrm{m} / \mathrm{h})\end{array}$ & $\begin{array}{c}\text { velocità media- } \\
2^{\mathrm{a}} \text { prova } \\
(\mathrm{m} / \mathrm{h})\end{array}$ \\
\hline $\mathrm{B} \rightarrow \mathrm{C}$ & 75 & 23.6 & 23,6 \\
\hline $\mathrm{C} \rightarrow \mathrm{D}$ & 75 & 19,6 & 25,0 \\
\hline $\mathrm{D} \rightarrow \mathrm{E}$ & 75 & 28,2 & 26,5 \\
\hline $\mathrm{E} \rightarrow \mathrm{F}$ & 50 & 33,3 & 28,5 \\
\hline $\mathrm{F} \rightarrow \mathrm{G}$ & 15 & 39,4 & 30 \\
\hline TOTALI & 290 & 25,8 & 25,8 \\
\hline
\end{tabular}

TABElla 2 - Andamento della velocità di arretramento nelle due prove (il punto $G$ coincide con la posizione dell'aspo e quindi con il fine corsa del carrello porta irrigatore).

TABLE 2 - Backward velocity during the tests.

\section{RISULTATI E DISCUSSIONE}

\section{Results and discussion}

Prima prova (senza regolarizzazione della velocità)

Sulla base dei dati della tabella 2 si può considerare la grande variabilità della velocità di avanzamento, che passa da un valore medio iniziale di $23,6 \mathrm{~m} / \mathrm{h}$ ad uno finale di $39,4 \mathrm{~m} / \mathrm{h}$, con un incremento circa del $70 \%$, determinato dalla progressiva diminuzione della forza d'attrito del tubo sul terreno e dall'aumento della velocità di avvolgimento del tubo sull'aspo in assenza del sistema di controllo.

In tali condizioni e come diretta conseguenza la distribuzione pluviometrica longitudinale mostra una estrema variabilità con un progressivo decremento delle altezze medie più evidente nel tratto dell'appezzamento a "regime" da C ad E .

L'estrema variabilità dei dati nelle diverse zone dell'appezzamento rende l'adacquamento estremamente disomogeneo con variazioni percentuali rispetto alla media superiori al $100 \%$ (fig. 2).

Per i dati relativi alla pluviometria nella zona all'inizio del percorso non è stato possibile differenziare il tempo di sosta $(t s)$ da quello di arretramento $(t a)$.

Per quanto riguarda l'andamento della pluviometria trasversale si fa notare come la distribuzione idrica sia quella tipicamente "a campana" con i valori massimi nella zona centrale di lavoro dell'irrigatore e i minimi verso la parte distale della gittata .

Questa variabilità, propria di ogni irrigatore, può essere ridotta con la sovrapposizione dei getti tra due postazioni adiacenti. Sovrapposizioni comunque sempre previste dai costruttori di rotoloni, la cui documentazione prescrive che tra due postazioni adiacenti la distanza sia pari all' $85 \%$ della larghezza teorica bagnata (pari a due volte la gittata).

Si può anche notare come nelle due zone di inizio e fine percorso corrispondenti agli andamenti A-B e F, l'effetto dell'adacquamento durante il tempo di sosta 
ts riduce sensibilmente la disuniformità della distribuzione sull'intero appezzamento. Occorre inoltre rilevare come, a causa della disuniformità distributiva in relazione alla variabilità della velocità di arretramento nella zona terminale (dove è massimo il valore della velocità) il tempo di sosta, calcolato rispetto alla velocità media, è risultato insufficiente. All'opposto, nella zona iniziale con una velocità molto inferiore alla media, il $t s$ calcolato è stato eccessivo in quanto ha comportato un valore idrico totale superiore alla media voluta.

La gittata media riscontrata (pari a $50 \mathrm{~m}$ ) corrisponde a circa il $10 \%$ in meno rispetto al valore dichiarato dalla ditta costruttrice. La pressione, sia alla turbina che all'irrigatore, si è mantenuta praticamente costante per tutta la durata della prova: alla turbina intorno a $0,8 \mathrm{MPa}$, all'irrigatore intorno a $0,4 \mathrm{MPa}$, valori simili a quelli dichiarati dalla ditta costruttrice.

Il vento si è mantenuto mediamente entro il valore di $5 \mathrm{~km} / \mathrm{h}$, passando da $1-2$ a $10 \mathrm{~km} / \mathrm{h}$ durante le prove; quindi mediamente di poco superiore al valore soglia di $4 \mathrm{~km} / \mathrm{h}$, oltre il quale il vento comincia ad incidere in maniera rilevabile sulla omogeneità della distribuzione [10]. La sua direzione, spesso rimasta nel senso longitudinale rispetto all'asse di lavoro del carrello, a volte ha modificato la direzione (W-NW) incidendo comunque poco sugli spostamenti della massa idrica distribuita.

I consumi di combustibile rilevati nelle prove sono stati dell'ordine di 12-13 1/ora, e quindi circa 300 litri durante l'orario di adacquamento (su 3 ettari di superficie), corrispondenti a 100 litri/ettaro.

Dato il prezzo del gasolio di $0,35 € / 1$, il costo per ettaro irrigato e per intervento è risultato di $35 €$. La manodopera impiegata, invece, è stata di circa 1,5 ore per postazione. Considerato un costo orario della manodopera aziendale di circa $13 €$, ogni postazione ha comportato una spesa di $20 €$, corrispondente ad un costo ad ettaro e per intervento pari a circa $7 €$.

L'intensità oraria di precipitazione è risultata pari a $11,5 \mathrm{~mm} / \mathrm{h}$. Tale valore, tenendo conto delle caratteri- stiche granulometriche del terreno (di tipo tendenzialmente argilloso) può considerarsi mediamente elevata, anche se non proibitiva.

La polverizzazione del getto calcolata ha fatto rilevare valori degli indici di efficienza e di polverizzazione rispettivamente pari a 1,25 e 1,45, corrispondenti ad una pioggia caratterizzata da gocce di elevato diametro, e che quindi sarebbe adatta solo per erbai e terreni decisamente sabbiosi. In questo caso la polverizzazione del getto andrebbe migliorata attraverso l'aumento della pressione o la riduzione del diametro del boccaglio in modo da ottenere un valore di Ip superiore a 2 . Tale grossolana polverizzazione, inadatta al tipo di terreno argilloso oggetto delle prove, è comunque non proibitiva rispetto alla sensibilità delle specifiche colture dell'azienda in studio (in particolare per il mais e specialmente nelle fasi fenologiche più avanzate).

\section{Seconda prova (velocità controllata)}

Sostanzialmente differenti sono i risultati ottenuti dalla prova con progressiva regolazione della velocità di avanzamento del semovente. In tali condizioni infatti, l'intervento manuale sul variatore di velocità ha contribuito a ridurre le variazioni della velocità del gruppo irrigatore: la velocità è infatti risultata compresa tra $23,6 \mathrm{~m} / \mathrm{h}$ e $30 \mathrm{~m} / \mathrm{h}$ sull'intero percorso, con un valore medio molto prossimo a quello desiderato di 25,6 m/h (tabella 2).

Diversamente della prova senza controllo della velocità, è stato possibile differenziare la distribuzione idrica anche nel tratto iniziale tra il tempo di sosta e quello durante l'arretramento. Di conseguenza sono risultati molto più uniformi i dati pluviometrici relativi allo sviluppo longitudinale, soprattutto nella zona centrale, nella quale l'altezza idrica media distribuita mostra scarti rispetto al valore medio assai modesti e ben si approssima al valore desiderato.

Per quanto concerne l'andamento della distribuzione idrica trasversale, esso presenta un diagramma "a campana". Evidentemente migliorando l'uniformità,

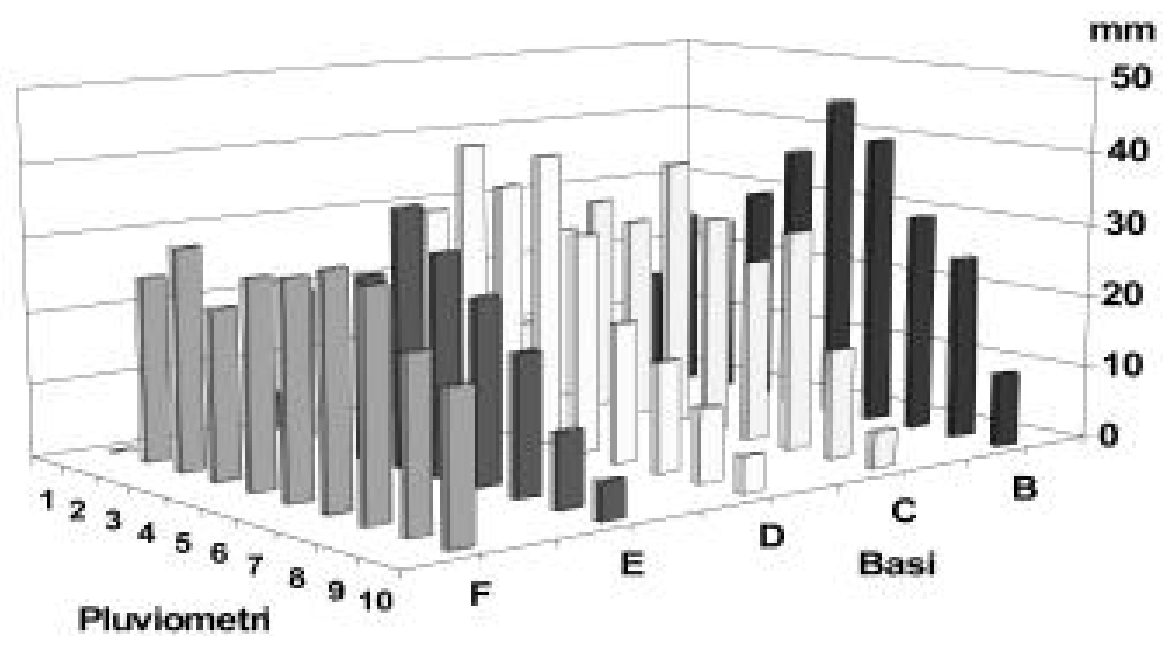

Fig. 3 - Andamento generale della pluviometria.

Fig. 3 - Overall value of rainfall. 


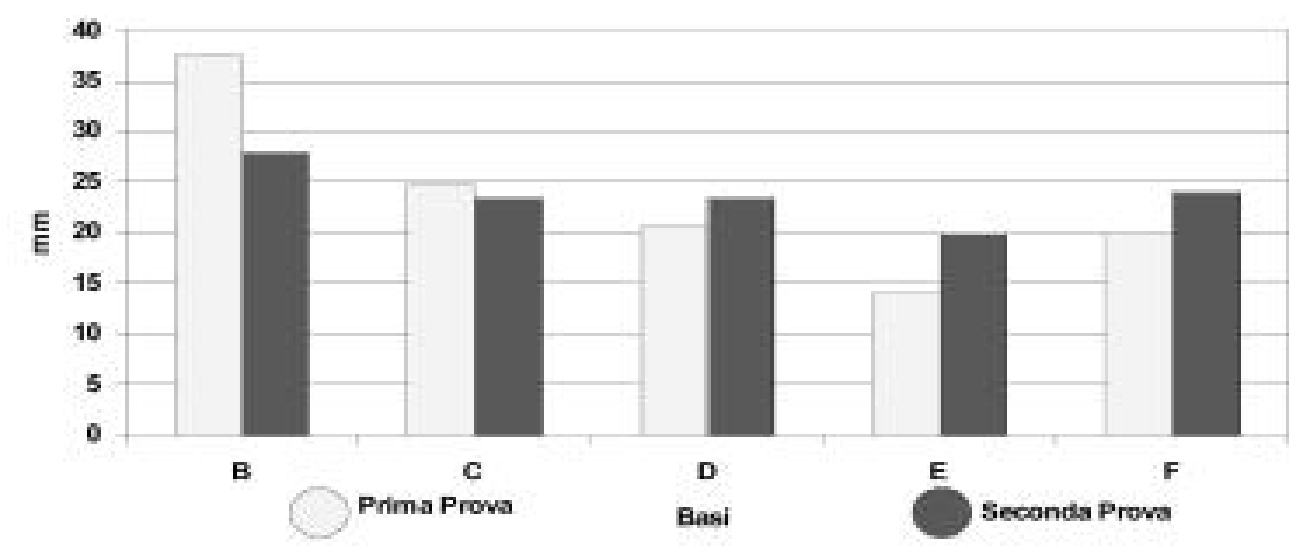

Fig. 4 - Confronto tra le pluviometrie medie longitudinali da B ad F. Prima e seconda prova.

Fig. 4 - Comparison of the longitudinal mean rainfall from B to F. First and second test.

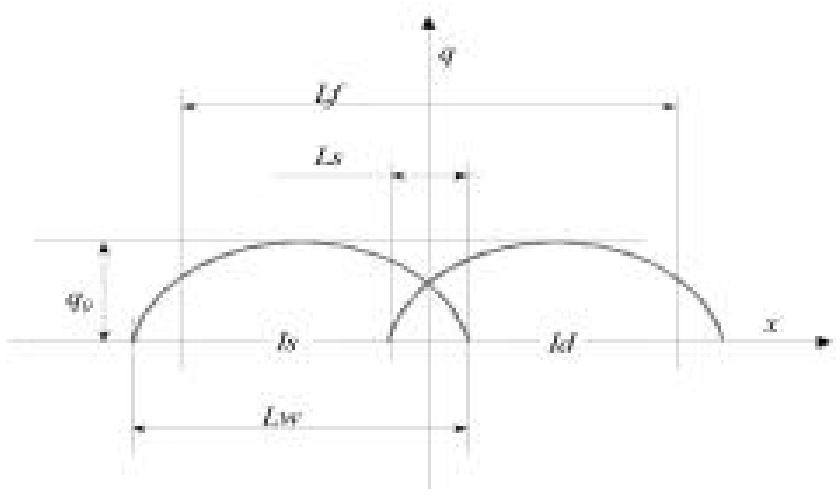

Fig. 5 - Schema di posizionamento degli irrigatori.

Fig. 5 - Sketch of the sprinkler positioning.

potrebbero essere necessarie sovrapposizioni delle gittate tra due adacquamenti adiacenti di minore entità. Ciò risulta evidente nella fig. 3 che rappresenta un andamento complessivo del "solido di bagnatura". In fig. 4 vengono inoltre confrontate le pluviometrie medie longitudinali delle due prove, in cui appare evidente la differente omogeneità distributiva.

\subsection{DeterminAZIONE DEL MIGLIORE POSIZIONAMENTO DEGLI IRRIGATORI}

\subsection{Determination of the best sprinklers positioning}

Alla luce dei dati sperimentali e delle considerazioni conclusive espresse al paragrafo precedente, si propone un modello di calcolo inedito sulla base del quale giustificare, mediante un esplicito criterio, il valore di sovrapposizione delle gittate suggerito dal costruttore. A tal fine si considera la semplice situazione schematizzata qualitativamente in figura 5 , dove un campo di larghezza $L f$ è servito con due irrigatori (Is e $I d)$, ciascuno dei quali bagna un'area di ampiezza $L w$, pari evidentemente ad doppio della gittata, secondo una distribuzione nota ma non uniforme. Dipendentemente dalla distanza tra gli irrigatori esisterà una zona di sovrapposizione tra le aree bagnate $(L s)$ : scopo del- lo sviluppo che segue è la determinazione della distanza tra i due irrigatori tale da garantire la minima disuniformità nel profilo di distribuzione dell'acqua complessivo.

Dai valori di pluviometria trasversale osservati su dieci sezioni trasversali (fig. 2 e fig. 3), si ricava un profilo di adacquamento medio, approssimabile con la semplice relazione polinomiale nel riferimento $(Q, x)$ di fig. 5: $Q(x)=-a x^{2}+b x+c$ con coefficiente di determinazione $\mathrm{R}^{2}>98 \%$ (fig. 6). Tale relazione può essere riscritta in riferimento agli irrigatori destro e sinistro, caratterizzando utilmente i parametri $a, b$ e $c$ per mezzo dei parametri operativi $L w, L s$ e $Q_{0}$ con le seguenti sostituzioni:

$$
\begin{aligned}
& \text { a) } Q_{0} \frac{{ }^{I s}}{L w \cdot k_{2}} \quad Q_{0} \frac{{ }^{l d}}{l w \cdot k_{2}} \\
& \text { b) }-Q_{0} \frac{4}{k_{2}} \quad Q_{0} \frac{4}{k_{2}} \\
& \text { c) } Q_{0} \frac{L s \cdot k_{1}}{L w \cdot k_{3}} Q_{0} \frac{L s \cdot k_{1}}{L w \cdot k_{2}}
\end{aligned}
$$

Essendo $k_{1}=2 L w-L s$ e $k_{2}=3 L w-2 L s$.

Con tali espressioni è possibile calcolare la quantità d'acqua distribuita complessivamente dai due irrigatori $(Q=Q s+Q d)$, in funzione della distanza dalla mezzeria $(x)$, della distanza tra gli irrigatori (espressa attraverso $L s$ ) e delle caratteristiche operative degli irrigatori stessi $\left(L w\right.$ e $\left.Q_{0}\right)$.

Se come indice di disuniformità nella distribuzione trasversale dell'acqua viene scelta la deviazione standard della funzione $Q(x)$ calcolata sul dominio $L f$ :

$$
\begin{aligned}
& S q=\sqrt{\frac{1}{L f} \int_{-L / 2}^{L / 2}(Q(x)-\overline{Q(x)})^{2} d x} \\
& \overline{Q(x)}=\frac{1}{L f} \int_{-i f / 2}^{4 / 2} Q(x) d x
\end{aligned}
$$

essendo allora la ricerca del valore minimo di $S q$ for- 


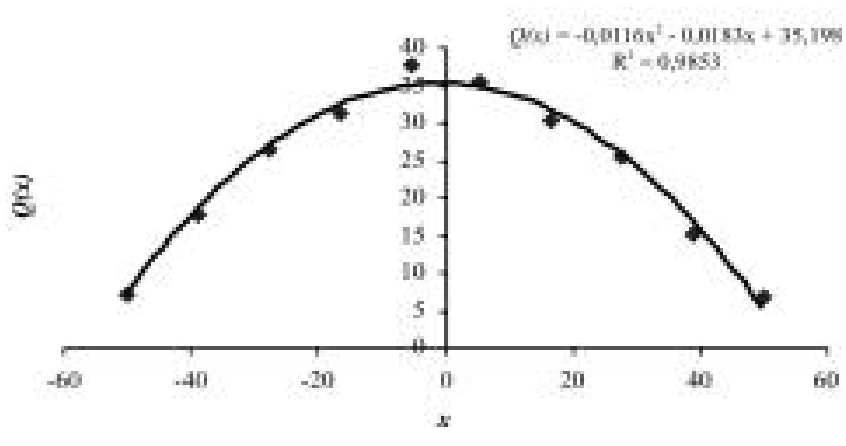

Fig. 6 - Interpolazione dei valori medi di pluviometria trasversale. Fig. 6 - Mean transversal rainfall values interpolation.

nisce la posizione degli irrigatori corrispondente alla distribuzione trasversale complessiva che più si avvicina a quella uniforme, sulla base dei valori funzionali relativi agli irrigatori in prova $Q_{0}$ ed $L w$.

In figura 7 sono riportati i valori calcolati di $S q^{*}$ $(S q$ rapportato al valore più alto tra quelli calcolati sull'intera area da irrigare) in funzione dei parametri adimensionali $L s^{*}=L s / L w$ ed $L f^{*}=L f /(2 L w)$, i quali si prestano alla seguente lettura:

$L f^{*}=1$, le due parabole coprono esattamente l'area da irrigare;

$L f^{*}<1$, le due parabole si possono sovrapporre e parte dell'acqua può cadere all'esterno dell'area da irrigare;

$L f^{*}>1$, parte dell'area più esterna non viene irrigata;

$L s^{*}=0$, sovrapposizione nulla (la distanza tra gli irrigatori è uguale alla gittata);

$L s^{*}=1$, sovrapposizione completa delle due parabole (la distanza tra gli irrigatori è uguale alla gittata).

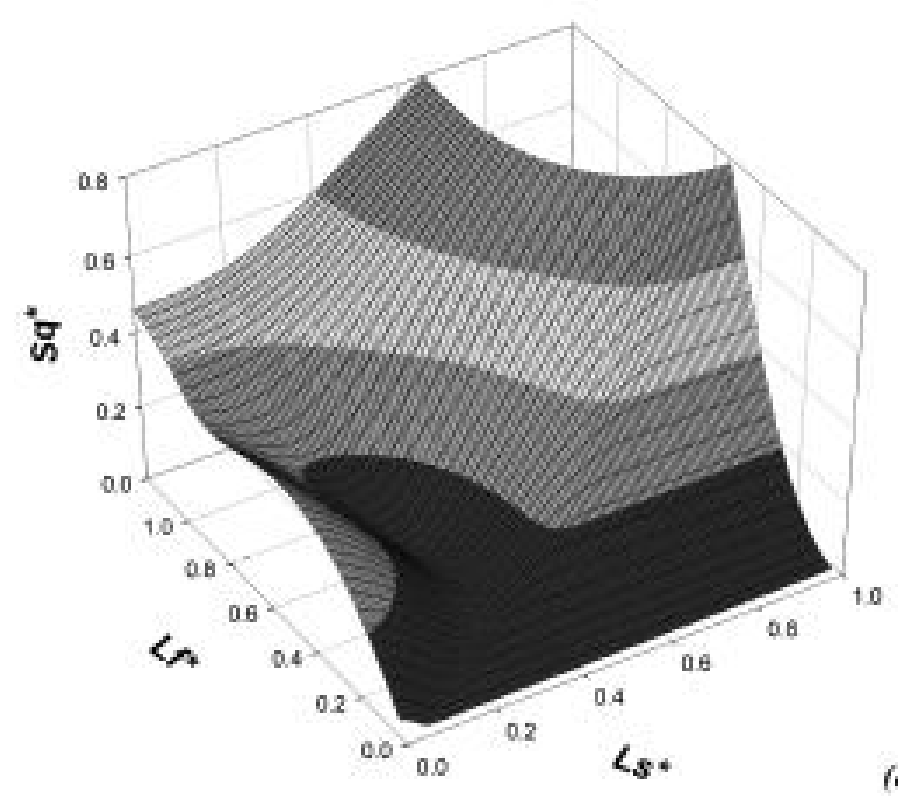

Per ogni valore di $L f^{*}$ (ovvero nota l'ampiezza dell'area da irrigare e dato un certo tipo di irrigatore, caratterizzato da $Q_{0}$ ed $L w$ ), il grafico di fig. 7 mostra che $S q^{*}$ ammette un minimo, in corrispondenza di un certo valore di $L s^{*}$. Tale minimo si sposta sui valori più bassi, all'aumentare di $L f^{*}$, fino ad azzerarsi per $L f^{*}>1$. Nel caso attuale, per i valori di $L f^{*}$ maggiormente ricorrenti, tale minimo si colloca nell'intervallo tra 0,1 e 0,3 e dunque, ricordando che l'interasse degli irrigatori vale $I=L w-L s$, si può concludere che la minima disuniformità si ha per valori di distanza tra gli irrigatori compresi nell'intervallo 0,7-0,9 Lw.

Tali considerazioni, oltre ad integrare la presente relazione, si proporrebbero come uno schema di calcolo generalizzabile che gli stessi produttori di irrigatori potrebbero utilizzare per corredare gli irrigatori di un nomogramma per il loro migliore piazzamento. Ciò potrebbe essere fatto senza eccessive difficoltà tenendo conto anche di situazioni più complicate rispetto a quella qui riferita come esempio, considerando più di 2 piazzamenti oppure distribuzioni d'acqua non simmetriche.

\section{CONCLUSIONI}

\section{Conclusions}

Le prove di campo hanno ancora una volta messo in evidenza aspetti sia positivi che problematici dei "rotoloni", in particolare:

- i dati tecnici di portata indicati dalla ditta costruttrice sono attendibili, mentre la gittata reale risulta inferiore di circa il $10 \%$ rispetto a quella indicata;

- l'uniformità distributiva dell'acqua in senso trasversale, rispetto all'avanzamento dell'irrigatore, è

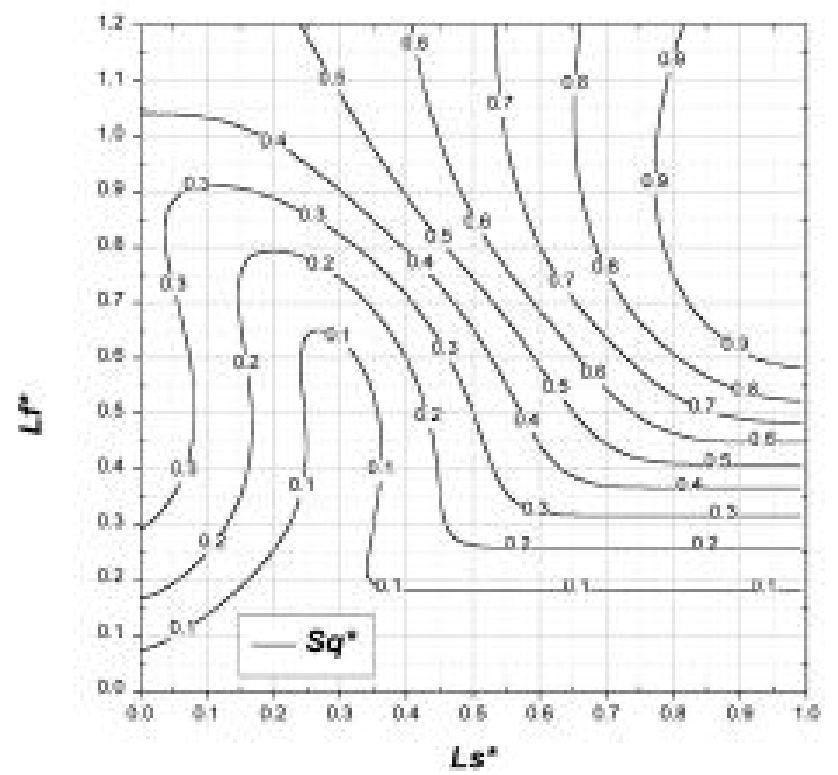

Fig. $7 a, 7 b$ - Deviazione standard della pluviometria trasversale in funzione del posizionamento degli irrigatori.

Fig. $7 \mathrm{a}, 7 \mathrm{~b}$ - Standard deviation of the transversal rainfall vs. sprinkler positioning. 
tipicamente "a campana" e quindi viene confermata la necessità di prevedere postazioni adiacenti, quando possibile, che consentano un'adeguata sovrapposizione del getto (distanza tra due postazioni pari all' $80-85 \%$ della larghezza bagnata);

- l'uniformità distributiva in senso longitudinale dipende dalla regolarità della velocità di lavoro e dall'erogazione iniziale e finale di un opportuno volume idrico con irrigatore non in movimento;

- con questi modelli di rotolone non provvisti di serie di un regolarizzatore della velocità di lavoro, tale parametro aumenta progressivamente durante il riavvolgimento del tubo, anche raddoppiando il proprio valore e quindi provocando un dimezzamento dell'altezza idrica media distribuita rispetto all'inizio del percorso. Rimane fondamentale quindi prevedere i regolarizzatori anche sui modelli di piccole e medie dimensioni;

- l'effetto battente sul terreno e sulle colture è in genere rilevante; l'aumento della pressione o la riduzione del boccaglio migliorerebbero tale parametro ma con inconvenienti legati alla necessità di adottare maggiori potenze e consumi o alla ridotta capacità operativa del semovente (riduzione della gittata e quindi aumento del costo orario di adacquamento e del numero di postazioni).

Si conclude che con i dovuti e possibili accorgimenti sopra evidenziati, questo semovente conserva comunque un grande interesse applicativo grazie ai suoi molti aspetti positivi.

\section{BIBLIOGRAFIA}

\section{References}

[1] Cemagref, CeP, Rned, Guide pratique Irrigation (1990), Imprimerie Landais, Noisy-le-Grand, France.

[2] GIOVAnardi R., Indagine conoscitiva sull'impiego degli irrigatori semoventi ad ala avvolgibile nel Veneto, L'Irrigazione (1982), XXIX, (3), 41-45.

[3] Giovanardi R., Ceccon P., Frisio S., Prove di funzionamento su irrigatori giganti semoventi (1985), L'Irrigazione, XXXII, (2), 9-16.

[4] Guidoboni G.,Anconelli S, Confronto economico tra diversi modelli di irrigatori semoventi(rotoloni) (1991), Supplemento a L'Informatore Agrario, XLVII, (19), 7-13.

[5] Merlo C., Macchine irrigue con tubazione avvolgibile e irrigatore rotante:l'offerta dei costruttori, $i$ criteri di scelta e d'impiego, Irrigazione e Drenaggio (2000), (2), 43-51.

[6] Taglioli G., I Semoventi irrigui ad ala avvolgibile, Genio Rurale (1984), n. 12, Edagricole, Bologna.

[7] Taglioli G., Semovente irriguo ad ala avvolgibile, Genio Rurale (1985), n.5, Ed agricole, Bologna.
[8] Taglioli G., Semoventi irrigui ad ala avvolgibile, Genio Rurale (1986), Ed agricole, Bologna.

[9] TAGLIOLI G., L'evoluzione del rotolone nell'irrigazione, L'informatore Agrario (2004), 19, Edizioni L'informatore Agrario spa, Verona.

[10] Taglioli G., Irrigazione e Drenaggio, Dispense per il Corso di Irrigazione e Drenaggio-Facoltà di Agraria Bologna.

\section{SUMMARY}

\section{HYDRAULIC TEST OF A SELF-PROPELLING SPRINKLER}

The correct management of the irrigation is a well known factor of success in agriculture, even as regard the water saving. As a consequence it was tested here a very common medium sized hose-reel irrigation machine, known in Italy as rotolone.

Several hydraulic and technical aspects were investigated, in two tests on a 3 ha field:

- the hydraulic distribution on the field, measured by means of 55 rainfall meter;

- the uniformity of the forward speed and the effectiveness of the controller;

- the effect of the standstill times on distribution quality;

- the hydraulic performance declared by the manufacturer;

- the water consumption, the manpower requirements;

- the quality of irrigation.

Moreover a theoretical analysis of the best overlapping between the range of two adjacent sprinkler was developed.

The tests have shown the importance of the regularization of the backward speed: in lack of this the variations can reach the value of $70 \%$.

The value of $85 \%$ in overlapping of wetted areas, recommended by manufacturers, was theoretically justified.

The measured mean range, at the recommended pressure, was $10 \%$ lower than declared by manufacturer. The rain hourly intensity was too high for the needing of the clay soil of the field. The jet spraying was coarse in relationship to the clay fraction of the soil but not for the crop (maize).

The hose-reel irrigation machine examined here, can maintain an high level of feasibility if some improvement were adopted.

\section{Key words:}

irrigation, self-propelling sprinkler, hydraulic performance. 
008_Taglioli(433)_57 28-01-2008 11:53 Pagina 64

$\phi$ 